\title{
Randomized controlled trial of pulmonary rehabilitation in severe chronic obstructive pulmonary disease patients, stratified with the MRC dyspnoea scale
}

\author{
J.A. Wedzicha*, J.C. Bestall+, R. Garrod*, R. Garnham*, E.A. Paul**, P.W. Jones ${ }^{+}$
}

Randomized controlled trial of pulmonary rehabilitation in severe chronic obstructive pulmonary disease patients, stratified with the MRC dyspnoea scale. J.A. Wedzicha, J.C. Bestall, R. Garrod, R. Garnham, E.A. Paul, P.W. Jones. (CERS Journals Ltd 1998.

ABSTRACT: This study tested the hypothesis that severity of respiratory disability may affect the outcome of pulmonary rehabilitation.

In this randomized, controlled study, 126 patients with chronic obstructive pulmonary disease (COPD) were stratified for dyspnoea using the Medical Research Council (MRC) dyspnoea score into MRC3/4 (Moderate) $(\mathrm{n}=66)$ and MRC 5 (Severe) dyspnoeic $(n=60)$ groups. The patients were randomly assigned to an eight week programme of either exercise plus education (Exercise group) or education (Control group). Education and exercise programmes for the moderately dyspnoeic patients were carried out in a hospital outpatient setting. Severely dyspnoeic patients were all treated at home. Those in the Exercise group received an individualized training programme.

There was a significant improvement in shuttle walking distance in the moderate dyspnoeic group, who received exercise training; baseline (mean \pm SEM) $191 \pm 22 \mathrm{~m}$, post-rehabilitation $279 \pm 22 \mathrm{~m}(\mathbf{p}<0.001)$. There was no improvement in exercise performance in the severely dyspnoeic patients receiving exercise. Neither group of control patients improved. Health status, assessed by the Total Chronic Respiratory Disease Questionnaire score, increased in the moderately dyspnoeic patients receiving exercise from $80 \pm 18$ to $95 \pm 17(p<0.0001)$ after rehabilitation. Much smaller changes were seen in the other three groups.

Improvement in exercise performance and health status in patients with chronic obstructive pulmonary disease after an exercise programme depends on the initial degree of dyspnoea.

Eur Respir J 1998; 12: 363-369.
*Academic Dept of Respiratory Medicine and **Dept of Environmental and Preventive Medicine, St Bartholomew's and Royal London School of Medicine and Dentistry, London Chest Hospital, London, UK. +Division of Physiological Medicine, St George's Hospital Medical School, London, UK.

Correspondence: J.A. Wedzicha Academic Dept of Respiratory Medicine

St Bartholomew's and Royal London

School of Medicine and Dentistry

London Chest Hospital

Bonner Road

London E2 9JX

UK

Fax: 441819832279

Keywords: Chronic obstructive pulmonary disease

dyspnoea

physical training

rehabilitation

stratification

Received: December 41997

Accepted after revision April 211998

Funded by a grant from the NHS Research and Development Programme on Physical and Complex Disabilities.
Chronic obstructive pulmonary disease (COPD) is an important cause of morbidity and mortality. It is characterized by progressive airflow obstruction [1] that leads to permanent disability and impaired mood state, especially with increasing severity of COPD [2]. Pulmonary rehabilitation programmes incorporating physical training have been advocated for these patients, with the aim of improving exercise capacity and quality of life [3-7].

A recent meta-analysis of rehabilitation showed that exercise training improves the patient's sense of control over their disease and reduces dyspnoea [8]. However, the benefit in terms of improvement in exercise capacity was not so clear after training, because the confidence interval of the effect size was wider than the minimum clinically important difference. The lack of a consistent improvement in exercise capacity between studies may have been due to heterogeneity in the level of disability between study populations or to the intensity of training programmes used.

Previous randomized controlled trials have included mainly patients with moderate COPD. No trials have specifically investigated patients with severe COPD who are housebound. These patients have considerable dyspnoea and fre- quent hospital admissions and may benefit from appropriately designed rehabilitation programmes. There is no information concerning the size of the treatment effect achieved in such patients or any comparisons with patients with less severe disability who are mobile outside the home.

This randomized controlled study has been designed specifically to investigate whether there is heterogeneity in the response to exercise training in severe COPD patients with different degrees of disability. A unique feature of this prospective study is that patients have been stratified according to the level of dyspnoea into moderately dyspnoeic and severely dyspnoeic groups, with comparable controlled interventions and identical outcome measures.

\section{Methods}

\section{Patient selection}

One hundred and thirty-eight patients with stable severe COPD (70 males, 68 females; median age 70 yrs (range 44-81)) were recruited sequentially from the respiratory 
outpatient clinics at the London Chest Hospital. The study protocol was approved by the East London and City Health Authority Ethics Committee and all patients gave written consent. Entry criteria included a history of COPD, (with forced expiratory volume in one second (FEV1) $<70 \%$ predicted), with $<15 \%$ reversibility to inhaled salbutamol $400 \mu \mathrm{g}$ and exercise capacity that was limited by dyspnoea. All patients had to show clinical stability for at least 3 weeks prior to recruitment, with no exacerbation over this period. Medical treatment was optimized prior to entry and was not changed during the rehabilitation programmes. Patients were excluded if they presented with unstable angina, peripheral vascular disease or joint-limiting mobility conditions, were unable to understand or complete questionnaires or had completed a previous pulmonary rehabilitation programme.

Of the 138 patients approached, 10 declined to take part in the study. The remaining 128 were stratified according to disability using the Medical Research Council (MRC) Dyspnoea score. The MRC score is a five-item questionnaire in which patients categorize their own level of disability. Patients who selected grade 1 or 2 , "Short of breath with strenuous exercise or when hurrying", were excluded from the programme as being too fit $(\mathrm{n}=2)$. Patients who selected either grade 3 "Walk slower than people of the same age on the level or stop for breath while walking at own pace on the level", or grade 4, "Stop for breath after 100 yards or after a few minutes on the level", formed the moderate dyspnoea group $(n=66)$. Those who selected grade 5, "Too breathless to leave the house", formed the severe dyspnoea group $(n=60)$.

Within the two groups, the 126 patients were randomized to either the exercise training group (Exercise), who received both exercise training and education twice weekly for 8 weeks, or the education group (Control), who received only education twice weekly for 8 weeks. The randomization was made in blocks of 8 and codes were held in sealed envelopes. In the moderately dyspnoeic (MRC grade 3/4) group, 33 patients were randomized to the Exercise group and 33 to the Control group and they received their treatment in a hospital outpatient setting. Of the house-bound severely dyspnoeic (MRC grade 5) patients, 30 were randomized to Exercise and 30 to the Control education sessions and were all treated by physiotherapists at home.

\section{Assessments}

Assessments were performed before and after the eight week rehabilitation programme.

\section{Lung function}

Spirometry measurements before and after administration of a bronchodilator were taken using a rolling seal spirometer (PK Morgan, Rainham, UK). Resting blood gases were measured from earlobe samples with the subject breathing room air for $20 \mathrm{~min}$ (Ciba-Corning 278 Blood Gas Analyzer; Medfield, MA, USA) [9]. Gas transfer (transfer factor of the lung for carbon monoxide $(T \mathrm{~L}, \mathrm{CO})$ and carbon monoxide transfer coefficient $(\mathrm{KCO})$ ) was measured using the single-breath method (PK Morgan).

\section{Exercise tolerance and breathlessness}

Exercise capacity was assessed using the Shuttle Walk Test (SWT), which is a maximal externally paced incremental exercise test [10]. Patients were requested to walk between two cones placed $10 \mathrm{~m}$ apart and the speed of the walk was increased by a small increment after each minute and the instructions were standardized from a tape recording. The end-point was determined when the patient was unable to maintain the required speed. All patients were asked to perform the test twice with a rest of at least 20 min between walks and the second test result was used for analysis. Oxygen saturation levels were monitored throughout the walk, using a pulse oximeter (Minolta Pulsox 7; AVL Instruments, Schaffhausen, Switzerland) and supplemental oxygen was provided on the second walk if an oxygen desaturation $<85 \%$ was observed.

\section{Health status assessment}

Health status measurement was made using the following questionnaires.

St George's Respiratory Questionnaire (SGRQ). The SGRQ is a valid and reliable measure of health status in patients with COPD [11]. It has been shown to be sensitive to changes in health status with treatment for COPD [12, 13]. It consists of 50 items with 76 weighted responses and three component scores: symptoms, activities and impacts (psychosocial dysfunction). A total score is calculated from all three components, with zero indicating no health impairment and 100 representing maximum impairment.

Chronic Respiratory Disease Questionnaire (CRQ). The CRQ also measures health status and was specifically designed for assessment of change [14]. It comprises four component scores: dyspnoea, fatigue, emotional function and mastery, measured on a seven-point Likert scale. The dyspnoea component of the questionnaire is individualized to five activities which cause dyspnoea and are assessed in order of importance and severity to the patient.

Extended activities of Daily Living assessment (EADL). The Nottingham (EADL) scale is a short, self-administered questionnaire consisting of 22 items divided into four sections: mobility, kitchen, domestic and leisure [15]. This scale has been shown to be capable of distinguishing between patients with different levels of disability as described by the MRC dyspnoea scale and to correlate well with severity of disease [16].

Hospital Anxiety and Depression (HAD) Score. The HAD score was used to assess levels of anxiety and depression. It consists of 14 items which produce separate scores for anxiety and depression [17]. A HAD score of Š11 indicates clinical cases of anxiety and depression.

\section{Rehabilitation programmes}

Exercise programme. The exercise programme consisted of unloaded upper and lower limb training. The same physiotherapist supervised home and hospital programmes, 
ensuring a similar exercise component for both MRC grade 3/4 and MRC grade 5 patients throughout the study. Exercises were based on the principle of body conditioning, with low-intensity repetitions of isolated muscle groups designed to minimize dyspnoea whilst improving peripheral muscle tone. The aerobic component of the exercise programme involved fast walking over a $10 \mathrm{~m}$ distance (with the instructions to walk "as though you are late for an appointment") and unloaded cycling on a cycle ergometer. Patients receiving the home exercise programme performed a similar set of exercises including fast walking at home, with the only exception being cycle work. All patients in the exercise training group, whether at hospital or in the home, performed the exercises for $1 \mathrm{~h}$, including rest periods as needed. Patients were encouraged to exercise unsupervised for $1 \mathrm{~h}$ daily, in between the sessions with the physiotherapist. Intensity was determined on an individual basis and self-limited by dyspnoea. Each patient continued exercising until breathlessness was perceived as moderate to relatively severe on the Borg scale.

The MRC grade 5 group included 10 patients who were on long-term oxygen therapy. They exercised using supplemental oxygen at a flow rate sufficient to maintain saturations $>85 \%$. In the MRC $3 / 4$ group, four patients who showed exercise oxygen desaturation were assessed for oxygen requirements during initial exercise sessions.

Education programme. The education programme was standardized for both the Exercise and the Control groups, and for patients seen at home or at hospital. Education sessions lasted for approximately $45 \mathrm{~min}$ and included topics on the anatomy and physiology of COPD, bronchodilator therapy and inhaler devices, nebulizer therapy, long-term oxygen therapy, smoking cessation, relaxation, chest clearance, breathing techniques, nutritional aspects and psychological problems associated with respiratory disability, travel with COPD, and when to call the doctor.

\section{Statistical analysis}

The study was designed to test the impact of initial dyspnoea levels on the effect of physical exercise training. For this reason, analysis was confined to those patients who completed $50 \%$ of the training sessions (i.e. eight). This rule was set a priori, before the study commenced. The primary outcome measure was shuttle walking distance. The secondary outcome measures were quality-oflife assessments. All data was normally distributed except for EADL scores. Two-way analysis of covariance (ANCOVA) was used to analyse data [18]. The two factors were: 1) level of disability (moderate or severe), and 2) randomized rehabilitation group (Exercise or Control). The covariate for each variable analysed was the baseline measure. Student's t-tests were performed to assess any differences at baseline and were also used as post hoc tests to determine in which groups changes had occurred. Relationships between measured variables were examined using the Pearson's product moment correlation. Treatment effects for measures of exercise performance and quality-of-life scores were calculated. The effect sizes were calculated by subtracting the Control group measurement after rehabilitation from the Exercise group measurement after rehabilitation. This difference was then divided by the pooled SD value of the post-intervention measure. Effect sizes for moderate and severe groups were calculated separately. Significance for all analyses was accepted at $\mathrm{p}<0.05$.

\section{Results}

After the eight week rehabilitation programme 109 patients completed a full assessment (fig. 1). Of the remaining 17 patients not assessed at 8 weeks, four patients attended fewer than eight out of 16 sessions and nine patients stopped attending after two or fewer rehabilitation sessions. One patient was injured and unable to exercise. Two patients died before the end of the eight week programme and one patient was unable to complete a walking test, but completed questionnaires. In the MRC grade 3/4 group, who had rehabilitation as hospital outpatients, the mean number of sessions attended was 14 out of a maximum of 16 sessions. For the home rehabilitation group, the mean number of sessions was 15 out of a maximum of 16.

\section{Comparison of disability groups}

The baseline characteristics for the two groups of patients are shown in table 1. Patients in the severely dyspnoeic housebound group had very low baseline exercise

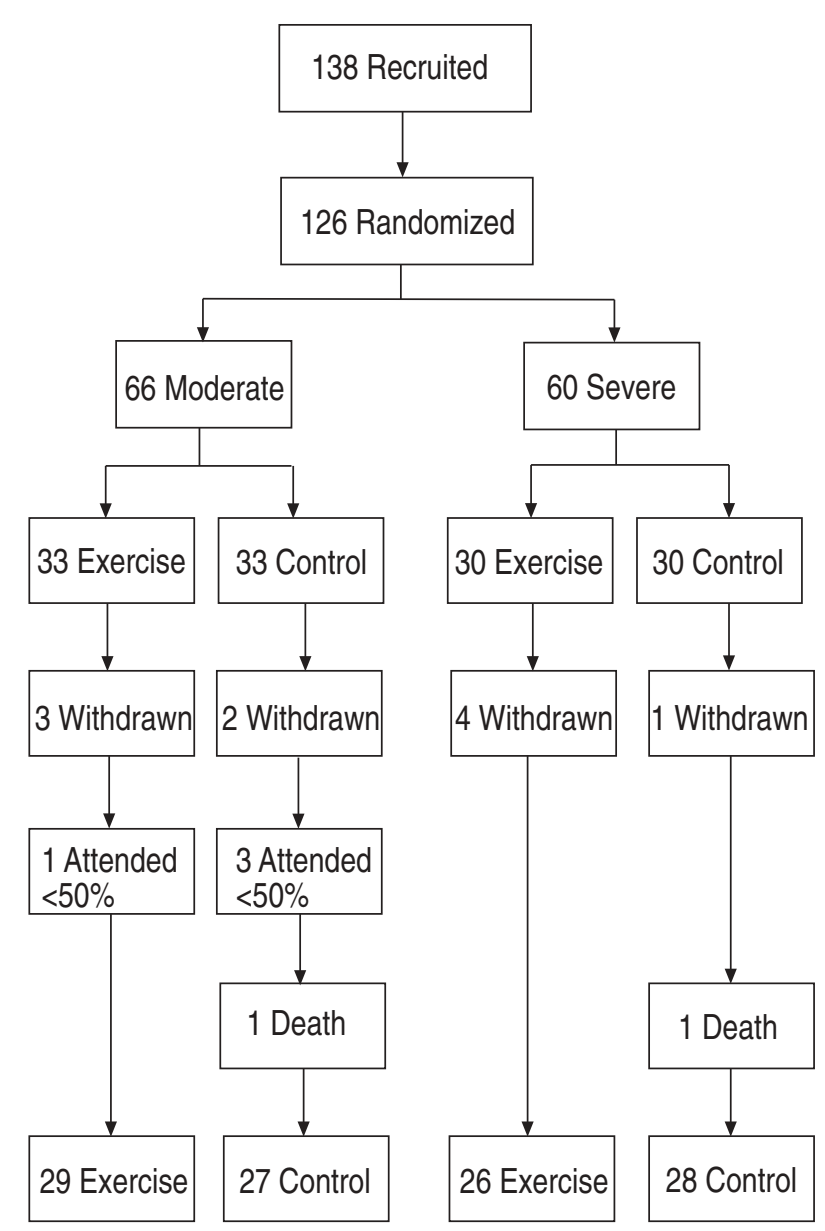

Fig. 1. - Trial profile. 
Table 1. - Baseline physiological parameters, exercise tolerance, health status and daily activity scores for moderate and severe groups

\begin{tabular}{|c|c|c|c|c|c|c|c|c|}
\hline \multirow[b]{2}{*}{ Baseline } & \multicolumn{4}{|c|}{ Moderate (MRC 3 and 4) } & \multicolumn{4}{|c|}{ Severe (MRC 5) } \\
\hline & $\begin{array}{c}\text { Exercise } \\
\mathrm{n}=29\end{array}$ & $\begin{array}{c}\begin{array}{c}\text { Control } \\
\mathrm{n}=27\end{array} \\
\end{array}$ & Diff. & $\overline{95 \% \mathrm{CI}}$ & $\begin{array}{c}\text { Exercise } \\
\mathrm{n}=26\end{array}$ & $\begin{array}{c}\text { Control } \\
\mathrm{n}=28\end{array}$ & Diff. & $95 \% \mathrm{CI}$ \\
\hline Age yrs & $68.6 \pm 8.9$ & $68.6 \pm 6.6$ & -0.02 & $-4.1-4.1$ & $73.0 \pm 5.9$ & $72.0 \pm 6.1$ & 1.0 & $-2.4-4.4$ \\
\hline FEV1 L & $0.95 \pm 0.32$ & $1.01 \pm 0.45$ & -0.06 & $-0.28-0.14$ & $0.87 \pm 0.41$ & $0.77 \pm 0.28$ & 0.1 & $-0.09-0.3$ \\
\hline FEV1 \% pred & $37 \pm 11$ & $38 \pm 12$ & 1.0 & $-8.3-5.1$ & $38 \pm 17$ & $36 \pm 12$ & 2.0 & $-6.3-11.2$ \\
\hline FVC L & $2.60 \pm 0.77$ & $2.55 \pm 0.71$ & 0.05 & $-0.4-0.5$ & $1.80 \pm 0.61$ & $1.85 \pm 0.72$ & -0.05 & $-0.4-0.3$ \\
\hline$P \mathrm{a}, \mathrm{O}_{2} \mathrm{kPa}$ & $8.94 \pm 1.06$ & $8.68 \pm 0.77$ & 0.26 & $-0.2-0.8$ & $8.26 \pm 1.64$ & $8.56 \pm 1.10$ & -0.3 & $-1.1-0.5$ \\
\hline$P \mathrm{a}, \mathrm{CO}_{2} \mathrm{kPa}$ & $5.80 \pm 0.54$ & $5.84 \pm 0.73$ & -0.04 & $-0.4-0.3$ & $6.17 \pm 0.92$ & $6.02 \pm 0.76$ & 0.15 & $-0.3-0.6$ \\
\hline $\begin{array}{l}T \mathrm{~L}, \mathrm{CO} \\
\quad \mathrm{mmol} \cdot \mathrm{min}^{-1} \cdot \mathrm{kPa}^{-1}\end{array}$ & $3.71 \pm 1.19$ & $3.75 \pm 1.62$ & -0.04 & $-0.8-0.7$ & $2.88 \pm 1.36$ & $2.67 \pm 1.42$ & 0.21 & $-1.88-2.29$ \\
\hline Shuttle m & $191 \pm 22$ & $217 \pm 22$ & -26 & $-84-39$ & $108 \pm 15$ & $75 \pm 11$ & 33 & $-4.7-71.7$ \\
\hline SGRQ Total & $50.6 \pm 13.7$ & $52.0 \pm 14.4$ & 2.4 & $-9.9-5.1$ & $63.7 \pm 13.9$ & $64.9 \pm 12.6$ & -1.2 & $-8.5-6.1$ \\
\hline CRQ Total & $80.3 \pm 18.1$ & $81.9 \pm 21.5$ & -1.6 & $-12-9$ & $70.0 \pm 17.1$ & $71.4 \pm 24.1$ & -1.4 & $13.2-10.3$ \\
\hline HAD Depression & $4.4 \pm 1.8$ & $5.2 \pm 3.9$ & -0.8 & $-2.4-0.8$ & $6.5 \pm 3.5$ & $7.1 \pm 2.5$ & -0.6 & $-2.3-1.03$ \\
\hline EADL* & $18(7-22)$ & $18(11-21)$ & - & - & $13(5-19)$ & $13(5-18)$ & - & - \\
\hline
\end{tabular}

Values are mean \pm SD. *: non-normal distribution scores, displayed as median (range). SGRQ: St George's Respiratory Questionnaire; CRQ: Chronic Respiratory Questionnaire; HAD: Hospital Anxiety and Depression Score; EADL: Extended Activities of Daily Living Score; MRC: Medical Research Council. FEV1: forced expiratory volume in one second; FVC: forced vital capacity; $P$ a, $\mathrm{O}_{2}$ : arterial oxygen tension; $\mathrm{Pa}_{\mathrm{a}, \mathrm{CO}_{2}}$ : arterial carbon dioxide tension; $\mathrm{TL}, \mathrm{CO}$ : lung carbon monoxide transfer factor; Diff.: difference; $\mathrm{CI}$ : confidence interval.

capacity, $90 \pm 10 \mathrm{~m}($ mean \pm SEM) compared with the moderate patients, $209 \pm 15 \mathrm{~m}(\mathrm{p}<0.0001)$. The severe group were also older $(\mathrm{p}<0.0009)$ and had worse health status scores (SGRQ Total scores $\mathrm{p}<0.0001$; CRQ Total scores $\mathrm{p}<0.006)$. They were also more impaired in terms of activities of daily living than the moderate group (EADL scores $\mathrm{p}<0.0001)$. However, the degree of airflow obstruction assessed by FEV1 was not significantly different between the two disability groups.

\section{Changes between treatment groups at baseline}

The baseline characteristics for the Exercise and Control groups for the two groups are shown in table 1 . There were no significant differences in baseline physiological characteristics between the Exercise and Control groups in either moderate (MRC 3/4) or severe (MRC 5) groups.

\section{Changes in exercise tolerance and pulmonary function}

Changes in shuttle walking distance before and after rehabilitation are shown in table 2 . There were significant differences in post rehabilitation exercise performance bet- ween the Exercise and Control groups after controlling for baseline exercise performance (ANCOVA main effects; $\left.\mathrm{F}_{1,104}=14.74, \mathrm{p}<0.0005\right)$. In addition, there was a significant interaction between the treatment group and the disability group in terms of their effect on the change in exercise performance $\left(\mathrm{F}_{1,104}=14.39, \mathrm{p}<0.0005\right)$. This indicates that the response to exercise training was affected by the initial MRC grade. To examine this further, post hoc analyses were performed to identify which group improved. These tests showed that only the moderately dyspnoeic patients in the Exercise group achieved a significant increase in exercise tolerance $(\mathrm{p}<0.001)$. There was no improvement in exercise performance in the severe patients who received exercise training. Neither group of control patients improved. There were no changes in spirometry or blood gases after the rehabilitation programmes in any of the four treatment groups.

\section{Health status}

There were no significant differences between the Exercise and Control groups for the SGRQ postrehabilitation total score controlling for baseline (main effect $F_{1,104}=2.31$,

Table 2. - Exercise performance assessed by shuttle distance and health status measurement assessed by St George's Respiratory Questionnaire (SGRQ) and Chronic Respiratory Questionnaire (CRQ) at baseline and postrehabilitation for moderate and severe disability groups

\begin{tabular}{|c|c|c|c|c|c|c|}
\hline Assessment & Disability & Group & Baseline & $\begin{array}{c}\text { Post- } \\
\text { rehabilitation }\end{array}$ & Change & $95 \% \mathrm{CI}$ \\
\hline \multirow[t]{4}{*}{ Shuttle walking distance $\mathrm{m}^{*}$} & Moderate & Exercise & $191 \pm 22$ & $279 \pm 22$ & 88 & $54-120$ \\
\hline & Severe & Exercise & $108 \pm 15$ & $118 \pm 15$ & 10 & $-6-27$ \\
\hline & Moderate & Control & $217 \pm 22$ & $201 \pm 21$ & -16 & $-46-14$ \\
\hline & Severe & Control & $75 \pm 11$ & $90 \pm 14$ & 15 & $-8-37$ \\
\hline \multirow{4}{*}{ SGRQ Total+ } & Moderate & Exercise & $51 \pm 13$ & $49 \pm 13$ & -2 & $-6-3$ \\
\hline & Severe & Exercise & $63 \pm 14$ & $66 \pm 14$ & 3 & $-2-7$ \\
\hline & Moderate & Control & $53 \pm 15$ & $57 \pm 16$ & 4 & $1-7$ \\
\hline & Severe & Control & $65 \pm 13$ & $67 \pm 11$ & 2 & $-1-5$ \\
\hline \multirow[t]{4}{*}{ CRQ Total+ } & Moderate & Exercise & $80 \pm 18$ & $94 \pm 16$ & 14 & $8-20$ \\
\hline & Severe & Exercise & $70 \pm 18$ & $74 \pm 21$ & 4 & $1-8$ \\
\hline & Moderate & Control & $82 \pm 22$ & $88 \pm 22$ & 6 & $1-10$ \\
\hline & Severe & Control & $71 \pm 24$ & $75 \pm 22$ & 4 & $1-8$ \\
\hline
\end{tabular}

*: mean \pm SEM; ${ }^{+}:$mean \pm SD. CI: confidence intervals. 
Table 3. - Treatment effects of exercise and control interventions for moderate and severe groups for measure of exercise performance and health status

\begin{tabular}{lccccc}
\hline Disability & $\begin{array}{c}\text { Outcome } \\
\text { measures }\end{array}$ & $\begin{array}{c}\text { Effect size } \\
\text { overall }\end{array}$ & $95 \%$ CI & $\begin{array}{c}\text { Treatment effect } \\
\text { natural units }\end{array}$ & $95 \%$ CI \\
\hline Moderate & Shuttle & 1.07 & $0.62-1.53^{* * *}$ & $104 \mathrm{~m}$ & $60-148^{* * *}$ \\
Severe & & -0.09 & $-0.63-0.45$ & $-4 \mathrm{~m}$ & $-31-22$ \\
Moderate & SGRQ Total & -0.52 & $-1.03-0.002$ & -5.4 & $-10.7-0.02$ \\
Severe & & 0.10 & $-0.44-0.66$ & 0.93 & $-3.9-5.8$ \\
Moderate & CRQ Total & 0.71 & $0.15-1.17 * * *$ & 8.9 & $2.1-15.8^{* * *}$ \\
Severe & & 0.02 & $-0.52-0.59$ & 0.23 & $-4.9-5.5$ \\
\hline
\end{tabular}

CI: confidence intervals; SGRQ: St George's Respiratory Questionnaire; CRQ: Chronic Respiratory Questionnaire. SGRQ scored in opposite direction to shuttle distance and CRQ. ${ }^{* * *}: \mathrm{p}<0.001$.

$\mathrm{p}=0.132$ ) and a weak interaction was found between treatment group and disability level $\left(\mathrm{F}_{1,104}=3.69, \mathrm{p}=0.057\right)$ (table 2). There were differences in the CRQ post rehabilitation total score between the Exercise and Control groups $\left(\mathrm{F}_{1,109}=3.89, \mathrm{p}=0.051\right)$. There was a weak interaction between the exercise programme and degree of disability $\left(\mathrm{F}_{1,104}\right.$ $=4.18, \mathrm{p}=0.043)$. Post hoc tests showed that the moderate group (MRC 3/4) that had received exercise showed the largest change in CRQ total score $(\mathrm{p}<0.0001)$. The moderately disabled control group and both treatment arms of the severely disabled group all showed smaller, but statistically significant improvements $(\mathrm{p}<0.05)$. There was no change in the EADL scores after rehabilitation in any group.

\section{Treatment effect size}

Effect sizes were calculated for measures of exercise performance and quality of life for the moderate and severe groups independently (table 3 ). In the moderate group, effect sizes for exercise performance and quality of life were moderate to large. In contrast, the effect sizes for the three other groups of patients were small.

\section{Correlations between exercise performance and quality of life}

At baseline, exercise performance was related to both health status measures. The correlation between exercise performance and SGRQ total score was $0.53(\mathrm{p}<0.0001)$ and between exercise performance and CRQ total score was 0.41 ( $p<0.0001)$. However, there were no relationships between changes in exercise tolerance and changes in either health status measure after the rehabilitation programme.

\section{Discussion}

Numerous studies have been performed on the effect of rehabilitation on exercise performance and quality of life in COPD [8]. The unique feature of the present study was stratification of the patients for severity of disability using the MRC Dyspnoea grade. Using this approach, it was shown that the effect of exercise training in COPD patients may depend on the initial level of dyspnoea. Patients with moderate dyspnoea (MRC grade 3/4), who were regularly mobile outside the home, showed quite large improvements in exercise capacity after physical training. In contrast, patients with severe disability (MRC grade 5), who were largely housebound owing to dyspnoea, showed no improvement in exercise performance following individu- alized physical training. The improvements in exercise capacity seen in the moderate patient group were similar to those achieved in other studies of outpatient pulmonary rehabilitation $[6,8,19]$.

The patients with moderate dyspnoea showed a greater improvement in health status after physical training than the other study groups. The size of these changes was similar to that reported from a meta-analysis of a number of trials in pulmonary rehabilitation [8]. The mean change in quality-of-life scores in the moderate group after exercise training was greater than the minimum clinically important difference for both the SGRQ and CRQ. There was no improvement in either exercise capacity or quality-of-life scores in patients with severe disability after an eight week training programme; however, there are few data in the literature against which to compare the results in this group of patients. Few randomized controlled trials of rehabilitation have included patients with equivalent levels of disability because such patients have considerable difficulty attending regular outpatient programmes. Two noncontrolled rehabilitation studies have suggested that patients with more impaired baseline exercise performance were likely to achieve greater percentage improvements in exercise performance after rehabilitation [20, 21]. However, the two studies described in the meta-analysis by LACASSE $e t$ al. [8], which showed the smallest change in exercise performance compared to controls after rehabilitation, recruited patients of equal severity to the present patient group, when assessed in terms of absolute FEV1 levels [22, 23].

The lack of improvement in exercise capacity or global increase in health status in the severely disabled housebound patients after exercise training may have been due to two factors. One may have been their very poor physical fitness and muscle deconditioning after many years of restricted activity. A study investigating training effects in patients with various levels of FEV1 showed that it was possible to achieve training effects in patients with marked impairment, although levels of disability were not assessed [24]. The second is that the exercise programme carried out at home was not sufficient to produce an improvement in physical performance. However, compliance with the home sessions was satisfactory in both exercise and control groups. It is possible that a longer and more intense exercise programme performed in a hospital setting with more intensive supervision would produce greater benefit, although most pulmonary rehabilitation studies have included training programmes of up to 8 weeks in duration, as in this study $[5,6]$. Patients with severe COPD develop significant anxiety and depression, especially with increasing hypoxaemia [2]. It is possible that these factors offset any 
benefit that could be achieved with physical training. Different rehabilitation strategies require evaluation in these severely disabled housebound patients, such as psychological and behaviour therapies which may improve functional exercise capacity.

Although exercise performance and health status improved in the moderate patient group, the mechanisms of these improvements are not clear. Training effects are unlikely to be the cause of the improvement in exercise performance, as the duration and intensity of the exercise programmes used in this study were similar to others that have been too low to achieve a true physical training effect $[25,26]$. Desensitization to dyspnoea has been suggested as an alternative mechanism to explain improvement in exercise performance after an exercise programme [26]. The impact of the exercise programme on health status in these patients appears to be nonspecific, with all modalities of the CRQ improving including emotions and mastery.

Patients with severe COPD become less mobile and reduce their activities of daily living. In a survey of patients with severe COPD on domiciliary long-term oxygen therapy, $50 \%$ of the patients did not leave the house and thus fell into the MRC dyspnoea group grade 5 and $78 \%$ were breathless walking around at home and performing daily activities [27]. Home rehabilitation programmes were performed in these patients because they were unable to cope with the exercise programme designed for more mobile patients and it enabled the physical training to be tailored to the individual needs and targets of each patient. This is the first randomized, controlled trial of physical training programmes that have been entirely performed in the patients' homes with physiotherapists visiting and supervising the patients at home. Although WIJKSTRA et al. [19, 28] referred to "home rehabilitation" in their study, the patients performed their exercise unsupervised at home and visited the physiotherapist twice weekly for training, with similar protocols to outpatient rehabilitation programmes.

The present findings emphasize the importance of encouraging exercise training at an earlier stage in the natural history of COPD, before the patient becomes severely disabled and housebound. Earlier onset of training may maintain the patient's mobility outside the home for a longer time, despite worsening lung function, although this specific point has not been formally studied. GoLDSTEIN et al. [5] studied a group of COPD patients with an FEV1 $<40 \%$ predicted and found improvements in exercise capacity and quality of life, though the rehabilitation programme was performed while patients were admitted as hospital in-patients. Such fully supervised programmes are expensive and facilities are not available in most countries for the prolonged admission required. However, it is possible that the degree of supervision during training plays a part during rehabilitation in severe patients and may have accounted for some of the differences between the results of the present study and those of other studies in patients with severe COPD.

This is the first study to examine the effects of exercise training as part of a comprehensive pulmonary rehabilitation programme in patients with severe chronic obstructive pulmonary disease, with a similar degree of airflow obstruction, but with different degrees of dyspnoea. The results of the study may explain the variable effects on exercise capacity with training that were reported in the meta-analysis by LACASSE et al. [8] and provide a basis for patient selection for rehabilitation. Patients who are candidates for rehabilitation programmes need to be selected on the level of their disability. There is a need for further study of the most effective forms of rehabilitation in chronic obstructive pulmonary disease patients with severe disability, who are frequent users of hospital and social service resources.

Acknowledgements: The authors are grateful to L.
John, Chief Respiratory Function Technician, London
Chest Hospital, and G. Foster for their assistance with the
study.

\section{References}

1. Fletcher $\mathrm{C}$, Peto R. The natural history of chronic airflow obstruction. BMJ 1977; i: 1645-1648.

2. Okubadejo AA, Jones PW, Wedzicha JA. Quality of life in patients with COPD and severe hypoxaemia. Thorax 1996; 51: 44-47.

3. McGavin CR, Gupta SP, Lloyd EL, McHardy GJR. Physical rehabilitation for the chronic bronchitic: results of a controlled trial of exercises in the home. Thorax 1977; 32: 307-311.

4. Cockcroft A, Saunders MJ, Berry G. Randomised controlled trial of rehabilitation in chronic respiratory disability. Thorax 1981; 36: 200-203.

5. Goldstein RS, Gort EH, Stubbing D, Avendano MA, Guyatt GH. Randomised controlled trial of respiratory rehabilitation. Lancet 1994; 344: 1394-1397.

6. Ries AL, Kaplan RM, Limberg TM, Prewitt LM. Effects of pulmonary rehabilitation on physiologic and psychological parameters in patients with chronic obstructive pulmonary disease. Ann Intern Med 1995; 122: 823-832.

7. Clark CJ. Is pulmonary rehabilitation effective for patients with COPD? Lancet 1996; 348: 1111-1112.

8. Lacasse Y, Wong E, Guyatt GH, King D, Cook DJ, Goldstein RS. Meta-analysis of respiratory rehabilitation in chronic obstructive pulmonary disease. Lancet 1996; 348: 1115-1119.

9. Pitkin AD, Roberts CM, Wedzicha JA. Arterialised ear lobe blood gas analysis: an underused technique. Thorax 1994; 49: 364-366.

10. Singh SJ, Morgan MDL, Scott S, Walters D, Hardman AE. Development of a shuttle walking test of disability in patients with chronic airways obstruction. Thorax 1992; 47: 1019-1024.

11. Jones PW, Quirk FH, Baveystock CM, et al. A self complete measure for chronic airflow limitation - the St George's Respiratory Questionnaire. Am Rev Respir Dis 1992; 145: 1321-1327.

12. Meecham Jones DJ, Paul EA, Jones PW, Wedzicha JA. Nasal pressure support ventilation plus oxygen compared with oxygen therapy alone in hypercapnic COPD. Am J Respir Crit Care Med 1995; 152: 538-544.

13. Jones PW, Bosh TK. Changes in quality of life in COPD patients treated with salmeterol. Am J Respir Crit Care Med 1997; 155: 1283-1289.

14. Guyatt GH, Berman LB, Townsend M, Pugsley SO, Chambers LW. A measure of quality of life for clinical trials in chronic lung disease. Thorax 1987; 42: 773-778.

15. Lincoln NB, Gladman JRF. The Extended Activities of Daily Living Scale: a further validation. Disability Rehab 1992; 1: 41-43.

16. Okubadejo AA, O'Shea L, Jones PW, Wedzicha JA. Home assessment of activities of daily living in patients with severe chronic obstructive pulmonary disease on 
long term oxygen therapy. Eur Respir J 1997; 10: 1572 1575 .

17. Zigmund AS, Snaith RP. The Hospital Anxiety and Depression Scale. Acta Psychiat Scand 1983; 67: 361-370.

18. Frison L, Pocock SJ. Repeated measures in clinical trials: analysis using mean summary statistics and its implication for design. Statist Med 1992; 11: 1685-1704.

19. Wijkstra PJ, van der Mark Th W, Kraan J, van Altena R, Koeter GH, Postma DS. Effects of home rehabilitation on physical performance in patients with chronic obstructive pulmonary disease. Eur Respir J 1996; 9: 104-110.

20. Niederman MS, Clemente PH, Fein AM, et al. Benefits of a multidisciplinary pulmonary rehabilitation program. Chest 1991; 99: 798-804.

21. Zu Wallack RL, Patel K, Reardon JZ, Clark BA, Normandin EA. Predictors of improvement in the 12-minute walking distance following a six-week outpatients pulmonary rehabilitation program. Chest 1991; 99: 805-808.

22. Booker HA. Exercise training and breathing control in patients with chronic airflow limitation. Physiotherapy 1984; 70: 258-260.
23. Jones DT, Thomson RJ, Sears MR. Physical exercise and resistive breathing in severe chronic airways obstruction are they effective? Eur J Respir Dis 1985; 67: 159-166.

24. Maltais F, Le Blanc P, Jobin J, et al. Intensity of training and physiologic adaptation in patients with chronic obstructive pulmonary disease. Am J Respir Crit Care Med 1996; 154: 442-447.

25. Belman MJ, Kendregan BA. Exercise training fails to increase skeletal muscle enzymes in patients with chronic obstructive pulmonary disease. Am Rev Respir Dis 1991; 143: 905-912.

26. Belman MJ. Exercise in patients with chronic obstructive pulmonary disease. Thorax 1994; 48: 936-946.

27. Restrick LJ, Paul EA, Braid GM, Cullinan P, Moore-Gillon J, Wedzicha JA. Assessment and follow up of patients prescribed long term oxygen therapy. Thorax 1993; 48: 708-713.

28. Wijkstra PJ, van Altena R, Kraan J, Otten V, Postma DS, Koeter GH. Quality of life in patients with chronic obstructive pulmonary disease improves after rehabilitation in the home. Eur Respir J 1994; 7: 269-273. 